Józef Młyński

Cardinal StefanWyszynski University in Warsaw, Poland

\title{
Caritas in Veritate - die Bedeutung von Liebe und Wahrheit für eine gute Gesellschaft
}

\author{
Caritas in Veritate \\ - Love and Truth an Implication \\ of a Good Society
}

\begin{abstract}
Love and truth are the two attributes of a well-functioning society. Unfortunately, these two basic values are often being devalued in the modern world. The reason for this is the lack of a sense of justice. Especially because in the social and cultural context the truth is often relativized. In this context it is worth analysing the Pope Benedict XVI's encyclical Caritas in Veritate, in which one should pay attention to a construction of the society in which today's human person lives.

Love and truth are the two attributes of a good society in with each human is equipped. From the very beginning, the human person is called to be directed by love and truth. He or she is called to this truth by his or her Christian life and ontic dimension of his or her nature. Society without love and truth will be threatened with agony, because it will not only stop developing but also its wrongly understood economical status will become a road to nowhere. That is why we should definitely accept the thesis that homo economicus must convert into homo religiosus in the name of love and truth, so that society will be good. Let love in truth leads to a good society, which the whole world is for us.
\end{abstract}

\section{Keywords}

Love, truth, justice, society. 


\section{Einleitung}

Die Liebe in der Wahrheit, die Jesus Christus mit seinem irdischen Leben und vor allem mit seinem Tod und seiner Auferstehung bezeugt hat, ist der hauptsächliche Antrieb für die wirkliche Entwicklung eines jeden Menschen und der gesamten Menschheit ${ }^{1}$. Diese Liebe ist die Kraft, die das Individuum dazu mobilisiert, sich aufopferungsvoll für Gerechtigkeit und Frieden zu engagieren, denn sie ist der einzige Weg zu einer angemessenen Entwicklung einer guten Gesellschaft.

Geht man den Worten der Enzyklika Caritas in veritate auf den Grund, so muss einem bewusst werden, dass die Kraft, die Stärke und der Träger des „Guten in der Gesellschaft“ Jesus Christus ist, der mit seinem Leben jedem Menschen zeigt, wie die Liebe in der Wahrheit gelebt werden kann. Das ist natürlich eine große Aufgabe, eine Aufgabe für das ganze Leben. Schon ganz am Anfang der menschlichen Existenz sollten wir feststellen, dass das Zentrum von allem die Liebe ist, die, so sagt der Papst, der Hauptweg der Soziallehre der Kirche ist ${ }^{2}$.

Es ist einfach festzustellen, dass die Verkündung der Liebe in der Wahrheit in den heutigen Zeiten nicht gerade einfach und problemlos ist. Diese Schwierigkeit wird impliziert durch die Schwierigkeiten eine objektive Wahrheit zu finden, die in unseren Zeiten häufig verfälscht und manipuliert wird. Deshalb zeigt der Heilige Vater auf eindeutige Art und Weise, dass es keinen anderen Weg als die Liebe in der Wahrheit gibt - eine besondere Aufgabe und Herausforderung für jeden Christen. Auf diesem Weg tauchen, wie wir wissen, Schwierigkeiten auf, entgegengesetzte menschliche Bestrebungen und ein gefälschtes Abbild der Wirklichkeit, das sich auf ihren verschiedenen Ebenen zeigt.

Trotz der vielen Schwierigkeiten: gesellschaftliche, ökonomische, kulturelle, auf die der zeitgenössische Mensch stößt, dürfen wir keine Angst haben, denn es ist unser aller Pflicht zu glauben, dass „... die Wahrheit ein Licht ist, das der Liebe Sinn und Wert verleiht. Es ist das Licht der Vernunft wie auch des Glaubens, durch das der Verstand zur natürlichen und

Vgl. Benedykt XVI., Caritas in veritate, 2009, Nr. 1.

2 Vgl. Benedykt XVI., Caritas in veritate, 2009, Nr. 2. 
übernatürlichen Wahrheit der Liebe gelangt: er erfasst ihre Bedeutung als Hingabe, Annahme und Gemeinschaft" ${ }^{\text {*3 }}$.

Im gegenwärtigen gesellschaftlichen und kulturellen Kontext wird die Wahrheit allzu oft relativiert, und gleichzeitig führt das Erleben der Liebe in der Wahrheit zum Verständnis, dass die Wahl christlicher Werte nicht nur eine sinnvolle Sache, nicht nur unerlässlich für den Aufbau einer ,guten Gesellschaft", sondern erforderlich ist für eine ganzheitliche Entwicklung des Menschen. In diesem Kontext lohnt es sich, eine Analyse der Enzyklika des Heiligen Vaters Benedikt XVI. zu unternehmen. Dabei ist auf das spezifische gesellschaftliche Konstrukt zu achten, in dem der zeitgenössische Mensch lebt. Dessen Koexistenz betrifft Probleme, mit denen der Mensch im Bewusstsein von Globalisierung und Pluralismus nicht zurecht kommt, denn die gefälschte Welt hat aufgehört, der Wahrheit und der Liebe angemessen Sinn zu geben. Möge die Übermittlung der Liebe in der Wahrheit Träger und Implikation einer guten Gesellschaft sein, die für uns die ganze Welt ist.

\section{Die Wahrheit über die Gesellschaft}

Der Begriff „Gesellschaft“ hat in den Sozialwissenschaften im Grunde genommen keine eindeutige theoretische Bedeutung. Ich werde mich seiner bedienen als bequeme Bezeichnung all jener Formen gesellschaftlichen Lebens, die der Kategorie des Individuums entgegengesetzt sind. Auf der einen Seite haben wir also: Gesellschaft, Bevölkerung, gesellschaftliche Gruppen, gesellschaftliches System, integrierte Gesamtheit, Institutionen, Gemeinschaften und Verbände, auf der anderen: das Individuum, das „Mitglied einer Gruppe, einer Gemeinschaft, einer Gesamtheit“ ist. Das Individuum nimmt eine Position im gesellschaftlichen System ein, ist angesiedelt in der gesellschaftlichen Struktur, spielt eine gesellschaftliche Rolle usw., es steht also in nicht zufälligen Beziehungen mit anderen Individuen im System. Die Sozialwissenschaften (von ihren philosophischen Ursprüngen über die Wissenschaften Soziologie und Ökonomie) leiden unter einem eigenartigen „ontologischen Dualismus“. Man nimmt häufig an, dass man das Funktionieren gesellschaftlicher Gruppen (das gesellschaftliche System) nicht aus der Beschreibung des Verhaltens der Individuen ableiten

\footnotetext{
3 Benedykt XVI., Caritas in veritate, 2009, Nr. 3.
} 
kann, dass die gesellschaftliche Gruppe „mehr“ ist als die Summe ihrer Teile, dass auf der überindividuellen Ebene eine gewissen „Logik“, „Dynamik“ des gesellschaftlichen Lebens zum Tragen kommt, die das Individuum zu einem bestimmten Verhalten zwingt (bewegt oder determiniert). Auf der anderen Seite argumentieren viele Wissenschaftler, dass diese „Gruppendynamik“eine Funktion (eine Resultante) des Verhaltens der Individuen, der individuellen Entscheidungen und Motivationen ist ${ }^{4}$.

Die Gesellschaft ist ein in Schichten, Klassen und Gruppen unterteiltes System. Anders ausgedrückt ist sie eine gesellschaftliche Hierarchie, eine Hierarchie der Eliten. Deshalb ist auch das erste Mittel der Untersuchung der Gesellschaft die Untersuchung ihrer Struktur. Darüber hinaus ist die Gesellschaft ein bestimmtes System der Arbeitsteilung, in dem das Individuum während seines ganzen Lebens eine bestimmte Aufgabe erfüllt. So steigert das Individuum seine Effektivität in Abhängigkeit davon, in welchem System es funktioniert. Zudem ist diese Gesellschaft eine gewisse Art von „demographischem Aggregat“, das sich auf bestimmte Art und Weise in der biologischen Reproduktion vollzieht. In der Gesellschaft handeln die Individuen: Durch das sogenannte gesellschaftliche Handeln werden sie zu einer Art von Schauspielern, Akteuren. Deshalb stellte M. Weber fest, dass gesellschaftliches Handeln die Grundlage der Soziologie bildet. Es beschreibt nämlich Verhaltensweisen, die übereinstimmen mit dem Sinn, den der Handelnde ihnen verliehen hat, jener Sinn markiert den Ablauf der interpersonellen Bezüge und hat einen subjektiven Einfluss auf das Netz der Vereinbarungen mit den anderen Individuen ${ }^{5}$.

Gesellschaftliches Handeln ist somit ein solches Handeln, das sich nach dem intentionalen Sinn des oder der Handelnden auf das Verhalten anderer Menschen bezieht und in seinem Ablauf auf diese hin ausgerichtet ist ${ }^{6}$. Deshalb nennt M. Weber die Teilnehmer an gesellschaftlichen Prozessen auch gesellschaftliche Akteure, die in bestimmten Verbindungen und Beziehungen mit anderen Teilnehmern dieses Prozesses verbleiben?

4 Vgl. J. Paleszczuk, Czym jest społeczeństwo, in: Wymiary życia społecznego. Polska na przełomie XXi XXI wieku, M. Marody (Hrsg.), Warszawa 2007, Verlag Scholar, S. 14.

5 Vgl. J. Szczepański, Socjologia, Rozwój problematyki i metod, Warszawa 1961, Verlag Żak, S. 330.

6 Vgl. M. Weber, Gospodarka i społeczeństwo, Zarys socjologii rozumiejacej, Warszawa 2002, Verlag PWN, S. 6.

7 Vgl. M. T. Frankowski, Socjologia. Makrostruktury społeczne, Warszawa 2008, Verlag M.M, S. 10. 
Auf das Konzept der gesellschaftlichen Akteure bezieht sich auch der amerikanische Soziologe J. Turner, der in seinem Werk „The Structure of Sociological Theory“ schreibt: „Die Analogie besteht damit zwischen den Akteuren auf der Bühne und den handelnden Menschen in der Gesellschaft. So wie Schauspieler genau festgelegte Rollen zu spielen haben, nehmen Individuen in der Gesellschaft festgelegte Positionen ein; so wie Schauspieler sich an das geschriebene Drehbuch halten müssen, so müssen die Handelnden in der Gesellschaft eine Norm befolgen; so wie Schauspieler den Anweisungen des Regisseurs Folge leisten müssen, so müssen die Individuen in der Gesellschaft Konformismus gegenüber denen zeigen, die Macht und Bedeutung haben; so wie Schauspieler gegenseitig auf die Art des Spiels des Partners auf der Bühne reagieren müssen, so müssen die Mitglieder der Gesellschaft ihre Reaktionen gegenseitig aneinander anpassen; so wie Schauspieler auf das Publikum reagieren, so spielen die Handelnden in der Gesellschaft ihre Rolle gegenüber verschiedenen Arten eines Publikums bzw. eines 'verallgemeinerten Anderen' und so wie Schauspieler aus vielfältigen Fertigkeiten und Fähigkeiten in jede Rolle ihre eigene, einzigartige Interpretation einbringen, so besitzen die Menschen in der Gesellschaft aus unterschiedlichen Konzeptionen ihrer selbst und unterschiedlichen Fähigkeiten zum Spielen einer Rolle ihre eigenen Interaktionsstile ${ }^{8}$.

Demnach durchläuft jeder Mensch in der Gesellschaft einen vielschichtigen Prozess der Aneignung zahlreicher Fähigkeiten und Anpassungen an das gesellschaftliche Leben. Der erste Prozess beginnt in der Ursprungs-Gruppe, der Familie. Das Individuum lernt in ihr zu sprechen und erlernt grundlegende kulturelle Normen und Werte. In der Soziologie nennt man diesen Prozess die Sozialisation', also die Vergesellschaftung. Im Grunde genommen ist die Sozialisation der erste Mechanismus kulturellen Einflusses auf das gesellschaftliche Leben des Menschen. Kurz gesagt ist die Sozialisation das

8 Vgl. J. Turner, Struktura teorii socjologicznej, Warszawa 1985, Verlag PWN, S. 419-420.

9 Den Begriff der Sozialisation führte F. Giddings 1897 in den Gesellschaftswissenschaften ein. Er beschrieb diesen Begriff damals als gesellschaftliche Entwicklung der menschlichen Natur oder des Charakters, also des gesellschaftlichen Zustandes der Psyche bei Personen, die sich in gegenseitigen Abhängigkeiten, in gegenseitigen Beziehungen befinden. Vgl. S. Kowalik, Szkic o koncepcjach socjalizacji, in: Edukacja wobec zmiany społecznej, J. Brzezinski, L. Witkowski (Hrsg.), Poznań-Toruń 1994, Verlag. Edytor, S. 277-291; W. P. Ogburn, W. F. Nimkoff, Handbook of Sociology, London 1947, S. 83, nach: M. Filipiak, Socjologia kultury, Zarys zagadnień, Lublin 2003, Verlag. UMCS, S. 90. 
Erreichen von Eigenschaften, die für den Menschen notwendig sind zum Leben in der Gesellschaft ${ }^{10}$ oder auch der Prozess feststehender Interaktionen ${ }^{11}$.

Unabhängig davon, auf welche Weise der Umfang der Beziehungen des Individuums gegenüber der Gruppe analysiert wird, bleibt immer ein Problem, das bestimmte Eigenarten der Form menschlichen Lebens betrifft. Immer werden die folgenden Fragen aufkommen: In welcher Gesellschaft lebt der zeitgenössische Mensch oder welche Gesellschaft entsteht nach all den Veränderungen und Verwandlungen, die mit der Industrialisierung und der Urbanisierung verbunden sind, schließlich welche Gesellschaft entsteht nach einer strukturellen Transformation? Ohne Zweifel kann man feststellen, dass das Ergebnis eine moderne, pluralistische Gesellschaft ist.

Dem gesellschaftlich-kulturellen Pluralismus entspricht ein struktureller Individualismus in der Einstellung und im Verhalten des Menschen. Einerseits haben wir es mit einem Prozess des Infragestellens von Werten und Normen, die allgemein verpflichtend sind, zu tun; auf der anderen Seite aber mit einer Zunahme individualisierter und subjektiv wichtiger Kriterien. In seiner extremsten Form kann sich dieser Typ individualisierter Wertevorstellungen zu einem praktischen Nihilismus wandeln, in dem jede sinnhafte Orientierung und ethische Entscheidung ihre verbindliche Kraft verloren haben. Es fehlt dann jegliche feste Hierarchie von Werten und Normen. Eine übertriebene Betonung der Person als Subjekt - so stellt die katholische Kirche fest - führt dazu, dass der Mensch sich in seinem Individualismus verschließt und bringt Personen hervor, die unfähig sind, wahrhaft menschliche Beziehungen zu knüpfen ${ }^{12}$.

${ }^{10}$ Die Soziologen unterscheiden zwei Arten der Sozialisation: die primäre und die sekundäre. Im Verlauf der primären Sozialisation entwickelt das Kind ein erstes Gefühl individueller Identität und, indem es sich als Familienmitglied wahrnimmt, von Gruppen-Identität. Es erwirbt Werte, Normen und Handlungsweisen, die in der gesellschaftlichen Umwelt wirken, in der es aufwächst. Sekundäre Sozialisation betrifft erwachsene Personen. Sie ist vor allem verbunden mit der Aneignung von Fähigkeiten, die zum Ausfüllen einer Rolle im Beruf, für ein Familienleben als Erwachsener und für die Partizipation am öffentlichen und politischen Leben notwendig sind. W. Modzelowski, Wprowadzenie do socjologii, Katowice 1999, Verlag BPS Śląsk, S. 70-71. Bildung für das Leben in der Familie ist lesenswert: J. Stala, Katecheza o matżeństwie i rodzinie w Polsce po Soborze Watykańskim II, Tarnów 2004, Verlag Biblos, S. 167-299; J. Stala, Katecheza rodzinna. Próba teologicznego zarysu zagadnienia, in: Rodzina. Bezcenny dar i zadanie, J. Stala, E. Osewska (Hrsg.), Radom 2006, Verlag Polwen, S. 187-195.

${ }^{11}$ Vgl. J. Kuplińska, Człowiek jako istota społeczna, in: Socjologia ogólna, Z. Krawczyk, W. Morawski (Hrsg.), Warszawa 1986, Verlag Znak, S. 14.

${ }^{12}$ Vgl. Johannes Paul II., Pastores dabo vobis, Nr. 7. 
Fakt ist, dass die Gesellschaft, in der wir leben, schnellem Wandel unterliegt und der grundlegende Kontext jenes Wandels der gesellschaftlich-kulturelle Pluralismus ist, der häufig schädliche Ideologien fördert: Schließlich ist jener kulturelle Pluralismus, so stellt J. Marianski fest, „der Verlust der absoluten Bedeutung von Werten und menschlichen Normen, insbesondere sofern sie mit der Religion verbunden sind. Über die akzeptierten Werte und Normen entscheidet letztlich das Individuum, das eher von den eigenen Möglichkeiten als von den ihm aufgezwungenen religiösen Denk- und Handlungsmustern geleitet wird. Das Individuum legt selbst nach seinen Bedürfnissen und Ambitionen Ziele und Mittel ihrer Realisierung fest (Moral wird für eine private Angelegenheit gehalten). Der Prozess der Individualisierung vertieft sich im Bereich der Möglichkeiten und Lebensperspektiven“113.

Die zeitgenössische Gesellschaft ist differenziert, charakterisiert von der gesellschaftlichen Trennung der Individuen, die sich ausdrückt in allerlei Arten von Ungleichheiten. Das Problem gesellschaftlicher Ungleichheiten gehört zu den wichtigen Fragen rationalen und objektiven Denkens und Empfindens jedes Menschen. Das ist so, weil man, wenn man die Schichtung der Gesellschaft untersucht, berücksichtigen muss, was mit den Individuen, die sich in einer gegebenen Gesellschaft befinden, passiert. Überaus häufig werden bei der Beschreibung gesellschaftlicher Ungleichheiten nur Unterschiede in der ökonomischen und beruflichen Stellung berücksichtigt und das konkrete Subjekt der Differenzierung vergessen, nämlich das Individuum. Es ist doch klar, dass sich alle menschlichen Gemeinschaften aus Individuen zusammensetzen, die sich voneinander unterscheiden: in der Größe, in der Haar- und Augenfarbe, im Gewicht und auch im Grade der Anfälligkeit für Krankheiten. Sie unterscheiden sich in Geschlecht und Alter, in Fähigkeiten und Fertigkeiten, auch im ökonomischen und gesellschaftlichen Status, im Umfang ihres Einflusses und in ihrem Ausbildungsniveau. Zwar könnte man den Katalog von Unterschiedsarten noch wesentlich verlängern, jedoch würde jede Aufzählung nur dazu dienen, sich der vielen Arten von Unterscheidungsmöglichkeiten menschlicher Individuen bewusst zu werden.

Vor allem anderen ist die zeitgenössische Gesellschaft materialistisch, tritt doch der Mensch selbst in ihr als Individuum auf, das auf dieser oder jener ökonomischen Ebene lebt. Gerade der „homo economicus“ bildet die

${ }^{13}$ J. Mariański, Młodzież między tradycja a ponowoczesnościa, Lublin 1995, Verlag KUL, S. 99. 
Gesellschaft, hat auf sie Einfluss und macht seine Position abhängig vom Erreichen von bestimmten Zielvorgaben, die nötig sind zu ihrem richtigen Funktionieren. Der ökonomische Mensch ist ein Mensch ohne jede Moral, ohne Moral, die ihm sozusagen einen Weg des richtigen Handelns aufzeigen könnte. Hat jedoch das zeitgenössische Individuum, das einzig und allein vom Niveau seines ökonomischen Status charakterisiert ist, eine Chance in dieser objektivierten gesellschaftlich-kulturellen Welt? Ja, wenn „haben“ sich in ,sein“ verwandelt, wenn nur das Individuum, das über das nötige ökonomische Kapital verfügt, die Prozesse der Selbstbestätigung erfährt.

\section{Liebe und Wahrheit als zwei Attribute menschlicher Existenz}

Alle Menschen haben in sich die Kraft zur authentischen Liebe. Jegliche Verantwortung sollte aus der Liebe geschöpft sein. Sie verleiht gewissermaßen der persönlichen Beziehung zu Gott und dem Nächsten einen wahren Gehalt, sie ist das Prinzip von Beziehungen in Freundschaft, Familien, kleinen Gruppen und auch das Prinzip der Beziehungen in gesellschaftlichen, wirtschaftlichen und politischen Zusammenhängen. Die Liebe ist das größte Geschenk, das Gott den Menschen gemacht hat, sie ist seine Verheißung und unsere Hoffnung ${ }^{14}$.

Der Papst richtet seine Aufmerksamkeit auf das oberflächliche Verständnis der Liebe, das keine ethischen Inhalte haben kann. Man kann die Liebe als authentischen Ausdruck des Menschseins erkennen wegen ihrer engen Verbindung zur Wahrheit. Nur in der Wahrheit kann die Liebe ehrlich, authentisch und von ganzem Herzen gelebt werden. Die Wahrheit ist der Antrieb für den Sinn und den Wert der Liebe. Das Licht der Wahrheit bezieht sich auf den Verstand und den Glauben. In einer Kultur ohne Wahrheit existiert das Risiko, die Liebe zu verderben. Sie wird Opfer der zufälligen Gefühle und Meinungen der Einzelnen, ein Wort, das missbraucht und verzerrt wird, bis es schließlich das Gegenteil bedeutet ${ }^{15}$.

Die Wahrheit erlaubt dem Menschen, objektiv zu handeln und der Liebe gemäß vorzugehen. Liebe, die der Wahrheit beraubt ist, entstellt die zwischenmenschlichen Beziehungen. Der Mensch sollte durch die Liebe das

\footnotetext{
${ }^{14}$ Vgl. Benedykt XVI., Caritas in veritate, 2009, Nr. 2.

15 Vgl. Benedykt XVI., Caritas in veritate, 2009, Nr. 3.
} 
Gute anstreben, sowohl das individuelle, als auch das gemeinsame Gute, das Gute für die ganze Gemeinschaft. Sich für das Gemeinwohl einzusetzen bedeutet, die Gesamtheit der Institutionen, die das soziale Leben rechtlich, sozial, zivil, politisch und kulturell strukturieren, einerseits zu schätzen und andererseits sich ihrer zu bedienen, so dass auf diese Weise die Polis, die Stadt, Gestalt gewinnt ${ }^{16}$.

Benedikt XVI. behandelt die Enzyklika Caritas in veritate wie die Fortsetzung der bisherigen Reflexion der Kirche über die Gesellschaft, vor allem in Bezug auf die historische Enzyklika Populorum progressio (1967) von Paul VI., die zum ersten Mal die kirchliche Soziallehre im weltweiten Kontext betrachtet. Sie beschäftigt sich mit den Bedingungen, die ein authentischer Fortschritt der Menschheit erfüllen soll, u. a. im Gegensatz $\mathrm{zu}$ einem materialistischen und reduktionistischen Fortschrittskonzept. Benedikt XVI. stellt fest, dass schon Paul VI. auf den weltweiten Horizont sogenannter gesellschaftlicher Fragen hinweist, zu denen die Kirche Stellung beziehen muss, während heute die ,gesellschaftliche Frage“ zu einer radikal „anthropologischen Frage“ geworden ist, die der absoluten Priorität des Menschen über Technik, Markt und Ökonomie Ausdruck verleiht. Die ganzheitliche menschliche Entwicklung setzt die verantwortliche Freiheit der Person und der Völker voraus: keine Struktur kann diese Entwicklung garantieren, wenn sie die menschliche Verantwortung beiseite lässt oder sich über sie stellt ${ }^{17}$. Jedoch führt jede Reflexion der kirchlichen Soziallehre auf die Begriffe Wahrheit und Liebe hin.

Es ist erforderlich, dass wir außer dem Anspruch auf eine freie menschliche Entwicklung auch auf ihre Wahrheit achten. Ein echter Fortschritt muss ganzheitlich sein. Der Papst erinnert somit daran, dass „ohne die Aussicht auf ein ewiges Leben dem menschlichen Fortschritt in dieser Welt der große Atem fehlt" ${ }^{\text {"18 }}$. Er fügt hinzu, dass Fortschritt, wenn er beschränkt bleibt auf Geschichte, Technik, Marktökonomie oder Sozialwissenschaften, dem Risiko ausgesetzt ist, dass er bloß auf den Slogan „mehr haben“ reduziert wird. In dieser Situation verlöre die Menschheit den Mut, bereit zu sein für die Annahme höherer Güter, für große und uneigennützige Initiativen, die der Liebe in der Welt entspringen. Paul VI., so schreibt Benedikt XVI., wünschte

\footnotetext{
${ }^{16}$ Vgl. Benedykt XVI., Caritas in veritate, 2009, Nr. 7.

${ }_{17}$ Vgl. Benedykt XVI., Caritas in veritate, 2009, Nr. 17.

${ }^{18}$ Benedykt XVI., Caritas in veritate, 2009, Nr. 11.
} 
sich, dass die wirtschaftliche Entwicklung ein reales Wachstum hervorrufen soll. Diese Entwicklung, dieses Wachstum haben Milliarden von Menschen aus dem Elend befreit. Man muss jedoch zugeben, dass diese wirtschaftliche Entwicklung durch Verzerrungen und drastische Probleme belastet war.

Der gegenwärtige Rahmen der Entwicklung ist polyzentrisch. Die Demarkationslinie, die reiche und arme Länder trennt, ist nicht mehr so deutlich wie zur Zeit von Populorum progressio. In Ländern, die zu den reichen gehören, bilden sich neue Formen von Armut. Auch deshalb ist gegenwärtig vor allem eine echte und ganzheitliche Entwicklung notwendig. „Das Heraustreten aus dem wirtschaftlichen Entwicklungsrückstand, ein an sich positives Faktum, löst nicht die komplexe Problematik der Förderung des Menschen: weder für die unmittelbar von diesem Fortschritt selbst betroffenen Länder, noch für die wirtschaftlich bereits entwickelten, und auch nicht für die noch armen Länder [...] $]^{\text {"19 }}$. Erst die Offenheit für das Leben ergibt eine echte Entwicklung, denn sie ,stärkt die moralischen Kräfte und befähigt zu gegenseitiger Hilfe. Wenn die reichen Völker die Offenheit für das Leben pflegen, können sie die Bedürfnisse der armen Völker besser verstehen, die Verwendung ungeheurer wirtschaftlicher und intellektueller Ressourcen zur Befriedigung egoistischer Wünsche bei den eigenen Bürgern vermeiden“20.

Der Mensch darf nicht egozentrisch und in sich selbst verschlossen in seinen Bedürfnissen und Zielen sein. Hedonismus ist verderblich und führt zum Bösen, also zur Sünde. „Die Überzeugung des Menschen, sich selbst zu genügen und in der Lage zu sein, das in der Geschichte gegenwärtige Übel allein durch das eigene Handeln überwinden zu können, hat den Menschen dazu verleitet, das Glück und das Heil in immanenten Formen des materiellen Wohlstands und des sozialen Engagements zu sehen“21. Denn der Mensch lebt nicht nur für sich selbst, sondern für die ganze Gesellschaft, für die Wirtschaft und das Einkommen der ganzen Gesellschaft, z. B. bei der Rente. Die Liebe vereint die Menschen auf reine Art und Weise, ohne Barrieren oder Grenzen. Die Einheit unter den Menschen entspringt aus den Worten Gott - Liebe. Um wahrhaftig menschlich zu sein, muss man selbstlos sein, das Ziel wirtschaftlichen Handelns muss das Erreichen des gemeinsamen Guten und vor allem die Sorge um die politische Gemeinschaft sein.

\footnotetext{
19 Benedykt XVI., Caritas in veritate, 2009, Nr. 23.

${ }^{20}$ Benedykt XVI., Caritas in veritate, 2009, Nr. 28.

${ }^{21}$ Benedykt XVI., Caritas in veritate, 2009, Nr. 34.
} 
Die ganze Menschheitsfamilie soll die notwendigen Ressourcen finden, um „mit Hilfe der Natur selbst, dem Geschenk Gottes an seine Kinder, und mit dem Einsatz ihrer Arbeit und ihrer Erfindungsgabe“"22, würdig zu leben. Von Generation zu Generation sollte die Erde in einem Zustand übergeben werden, der das Fortleben auf ihr erlaubt. Die Beziehung des Menschen zur Umwelt spiegelt wider, wie er andere Menschen behandelt und von ihnen behandelt wird. Das ist ein Signal für die Gesellschaft, ihren Lebensstil ernsthaft ökologischer auszurichten. Man darf sich nicht von reinem Konsumptionismus leiten lassen oder sich dem Hedonismus hingeben. Ein Wandel der menschlichen Mentalität ist erforderlich, damit die Gesellschaft besser und angemessen funktionieren $\mathrm{kann}^{23}$. Liebe und Wahrheit sind nicht nur Träger der menschlichen Existenz, sondern vor allem ein wichtiges Kriterium für eine Zivilisation der Liebe. Jede Liebe nämlich, jedes Kennenlernen lehrt uns etwas zu empfinden, was neu ist und es objektiv mit den Kriterien der Wahrheit zu interpretieren.

\section{Liebe und Wahrheit als zwei Dimensionen der Gesellschaft}

Für eine sich gut entwickelnde Gesellschaft sind gute Bürger, die ihr Leben von Liebe und Wahrheit leiten lassen, erforderlich. Papst Benedikt XVI. schreibt über die ganzheitliche Entwicklung des Menschen und damit über ihre beiden, komplementären Ebenen; er stellt nämlich fest, dass Liebe und Wahrheit der „hauptsächliche Antrieb für die wirkliche Entwicklung eines jeden Menschen und der gesamten Menschheit" sind. Schon in seinen ersten Sätzen stellte der Papst fest, dass Liebe und Wahrheit, so kann man sagen, die Kardinaltugenden in der Entwicklung des Menschen und der Gesellschaft sind. Der Mensch ist ein Teil des Systems, deshalb garantiert seine richtige Entwicklung die richtige Entwicklung des Systems. Liebe sollte wahrhaftig erlebt werden, gemäß der göttlichen Offenbarung und nach dem wichtigsten Gebot, das Gott als Gebot der Liebe aufgestellt hat - die Wahrheit. Die Liebe

${ }^{22}$ Benedykt XVI., Caritas in veritate, 2009, Nr. 50.

${ }^{23}$ Die Kirche ruft dazu auf, die Luft, das Wasser und vor allem die Erde zu schonen. Der Mensch muss den Begriff der Ökologie verstehen und ihn im Leben umsetzen können. „Die Beschädigung der Natur hängt nämlich eng mit der Kultur zusammen, die das menschliche Zusammenleben gestaltet. Wenn in der Gesellschaft die ,Humanökologie' respektiert wird, profitiert davon auch die Umweltökologie“. Benedykt XVI., Caritas in veritate, 2009, Nr. 51. 
ist die motivierende Kraft zum mutigen und aufopferungsvollen Engagement für Gerechtigkeit und Frieden, das unerlässlich ist für das Schaffen und das Funktionieren einer Gesellschaft. Es ist dies eine Kraft, die ihre Quelle in Gott hat - „,im Gott der ewigen Liebe und absoluten Wahrheit“"24. Liebe, Gott und Wahrheit sind die Fundamente jeder Gemeinschaft. Dort, wo Gott ist, ist auch die Liebe, denn Gott, der die Wahrheit ist, hat alles mit Liebe erschaffen und den Menschen zur Liebe berufen, damit er sie auf der Erde Wirklichkeit werden lässt.

Liebe und Wahrheit sind das Rückgrat der Gesellschaft und miteinander eng verbunden; diese beiden Kräfte geben den Menschen Kraft zum Leben. Die Liebe ist das grundlegende Prinzip aller Beziehungen: zu Gott, zum Nächsten, zum Freund, zur Familie, zur Gesellschaft, denn „Gott ist die Liebe“ ${ }^{\text {25 }}$, deshalb ist die Liebe alles. Die Liebe wiederum schöpft ihren Sinn und ihren Wert aus der Wahrheit, ohne die Wahrheit wäre sie leer, sie kann dann verdorben, auf reine Körperlichkeit reduziert und der echten Beziehung zum Nächsten beraubt werden. Papst Benedikt XVI. betont, dass die Liebe in der Wahrheit, die Wahrheit in der Liebe unverzichtbar sind für das Schaffen einer guten Gesellschaft und für die richtige und ganzheitliche Entwicklung der Menschheit ${ }^{26}$.

Eine wichtige Eigenschaft einer guten Gesellschaft ist das gemeinsame Gute, das aus dem Guten einzelner Personen besteht. „Jemanden zu lieben, das heißt jemandem das Gute zu wünschen und sich wirksam für dieses Ziel zu engagieren“, so lautet eine der Anforderungen von Liebe und Gerechtigkeit. Jeder Christ ist zur Nächstenliebe aufgerufen, und somit zur Achtung seiner Rechte und Pflichten, seiner Würde, seiner Freiheit und dessen, was immer er auch tut, er sollte immer auf das Gute des anderen, das eigene Gute, das gemeinsame Gute Rücksicht nehmen.

${ }^{24}$ Benedykt XVI., Caritas in veritate, 2009, Nr. 1.

25 1. Joh. 4,16 .

${ }^{26}$ Vgl. Benedykt XVI., Caritas in veritate, 2009, Nr. 1. Zu lieben heißt, dem Nächsten etwas zu schenken. Gerechtigkeit heißt, das hinzugeben, was einem anderen zusteht und ist somit die nächste Bedingung einer guten Gesellschaft. Der Grundsatz der Gerechtigkeit ist die Wahrnehmung des Guts, wie es einer Person zusteht. Der Mensch ist ein souveränes Subjekt und von daher steht ihm etwas zu, er hat auf etwas ein Anrecht. Die Aufgabe der Tugend der Gerechtigkeit ist, in uns den dauerhaften und unbeugsamen Willen auszubilden, das hinzugeben, was anderen von unserer Seite aus zusteht. Vgl. P. Jaroszyński, Etyka - dramat życia moralnego, in: Wprowadzenie do filozofii, M. Krąpiec (Hrsg.), Lublin 1996, Verlag KUL, S. 563. 
Die Gesellschaft wird von Personen geschaffen, die aufgerufen sind, zu ihrer Entwicklung beizutragen; aber sie bewältigen diese Aufgabe zuerst durch ihre eigene Entwicklung, zu der der Mensch berufen ist. Diese Entwicklung, diese Berufung hat auf der einen Seite eine transzendente Aufforderung als Quelle, auf der anderen Seite wird sie vom Menschen selbst unternommen als immanentes Wirken Gottes und gleichzeitig bedeutet sie eine Zweckmäßigkeit bzw. einen Sinn des Lebens. Solch eine Berufung ist eine Aufforderung, auf die es eine freie und verantwortungsvolle Antwort geben muss, sie begründet eine ganzheitliche menschliche Entwicklung und betrifft sowohl die natürliche als auch die übernatürliche Ebene ${ }^{27}$. Diese Entwicklung ist auf den Schöpfer ausgerichtet, auf die Wahrheit und das Gute; die Harmonie der menschlichen Natur ist für eine höhere Würde bestimmt, die der Mensch durch Christus erhält und die er zur Gänze durch einen „Humanismus, der seine Natur übersteigt und die größte Fülle des Lebens gewährt" erreichen kann. Die ganze Entwicklung einer Person, einer Gesellschaft muss auf Gott gegründet sein, der die Wahrheit und die Liebe ist, denn dank Ihm erkennen wir die Ordnung, ohne Ihn können wir uns verlieren und das Ziel und das Gute hören auf, Werte zu sein.

Die Entwicklung der Welt schreitet mit ungeheurem Tempo voran. Wir beobachten die Entwicklung der Wirtschaft, der Politik, vieler Wissenschaften: der Medizin, der Ökonomie, der Technik. All das erlaubt dem Menschen, besser zu leben, zu funktionieren. Aber ist alles auch gut für den Menschen? Ist der ganze Fortschritt gut? Nimmt die Entwicklung Rücksicht auf die Person, ihre Würde und ihre Rechte? Die derzeitige Gesellschaft unterscheidet sich deutlich von früheren Gemeinschaften. Gegenwärtig spielen bei den verschiedensten Tätigkeiten Technik, Technologie, Cyberwelten usw. die Hauptrolle. Als Resultat dieser „Maschinenzivilisation“ verarmen die menschlichen Beziehungen. Gleichzeitig sollten sich die menschlichen Beziehungen und Interaktionen in einer gut funktionierenden Gesellschaft erweitern. Eine gute Gesellschaft, die auf der Liebe und der Wahrheit gegründet ist, ruft auf zur Entwicklung eines Menschen, der den Kontakt zum anderen Menschen braucht, um sich zu entfalten und sich zu sozialisieren.

Die „Maschinenzivilisation“ hat bewirkt, dass der Mensch in Vergessenheit geraten ist und die menschliche Person bei den gesellschaftlichen Bedürfnissen nur noch eine Nebenrolle spielt. Die Rechte, die Papst Johannes XXIII.

\footnotetext{
27 Vgl. Benedykt XVI., Caritas in veritate, 2009, Nr. 18.
} 
unterstrichen hat, werden nicht so respektiert, wie sie respektiert werden müssten. Im Beruf ist der Mensch durch Maschinen ersetzt worden, was dazu geführt hat, dass viele Menschen ihre Arbeit verloren haben. Das Fehlen von Arbeit führte wiederum zu ungünstigen Konsequenzen im gesellschaftlichen Leben: die Familie wurde geschwächt, es entstand ein tiefgreifender Prozess der Migration ins Ausland, zu dessen Konsequenzen auch Scheidungen und mit der Ehe konkurrierende alternative Formen des Zusammenlebens gehören. Es folgte eine Erschütterung der Wertehierarchie, die uns von Gott gegeben ist und von der Lehre der Kirche unterstrichen wird. Man vergisst, dass ,,doch der Mensch Urheber, Mittelpunkt und Ziel aller Wirtschaft ist ${ }^{\text {"28 }}$. Papst Benedikt XVI. appelliert an die Regierungen der Welt, dass das erste Kapital, das man bewahren und schätzen muss, der Mensch ist - die Person, ihre Würde und Rechte. Der Mensch baut die Gesellschaft und deshalb wird die Sorge um sein Wohl der Gesellschaft zu Gute kommen. „Auch im Wirtschaftsleben sind die Würde der menschlichen Person und ihre ungeschmälerte Berufung wie auch das Wohl der gesamten Gesellschaft zu achten und zu fördern“29.

Es besteht kein Zweifel, dass Fortschritt aufgrund der wachsenden Bedürfnisse des Menschen notwendig ist und auch dazu, um ihm das Funktionieren zu ermöglichen. Die Entwicklung hat ihre Gesetzmäßigkeiten und Methoden, nach denen sie funktioniert, muss aber ,im Rahmen der sittlichen Ordnung so [sein], dass das verwirklicht wird, was Gott mit den

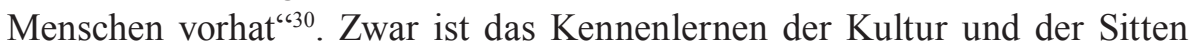
eines anderen Landes positiv, jedoch hat kulturelle Homogenisierung zu einem Verblassen der Bedeutung der Kultur einzelner Länder geführt, da die Kultur einzig und allein aufFakten reduziert wird. Diese Homogenisierung führt dazu, dass der Mensch aufgehört hat, sich in den Grenzen des Anstandes und der Kultur zu verhalten. Wenn wir z. B. an freie Beziehungen oder homosexuelle Beziehungen, Experimente am menschlichen Leben, Abtreibungen und viele andere beunruhigende Phänomene denken, dann müssen wir sagen, dass sie nicht nur eine Ausgeburt menschlichen Denkens sind, sondern auch aus anderen Kulturen stammen. Auf diese Art und Weise ist die Menschheit der Gefahr des Zwangs und der Manipulation ausgesetzt. Jedes Handeln, das mit Entwicklung, Fortschritt, dem Kennenlernen anderer Kulturen verbunden ist,

\footnotetext{
${ }^{28}$ Gaudium et Spes, Nr. 63.

29 Gaudium et Spes, Nr. 63.

${ }^{30}$ Gaudium et Spes, Nr. 64.
} 
muss sich auf konkretes Wissen stützen, auf wahrhaftige Erkenntnis. Deshalb benötigen wir gegenwärtig jene interdisziplinäre Sicht der Wissenschaft, um die Liebe in der Wahrheit, auf die sich eine gute Gesellschaft stützt, richtig erkennen zu können. Denn die Liebe benötigt Wissen, damit sie verbreitet und von innen belebt wird.

Der nach dem Bilde Gottes geschaffene Mensch ist ausgestattet mit einer transzendentalen Würde und dem Wunsch, „mehr zu sein“. Seine Natur bestimmt ihn dazu, über sich selbst hinaus zu wachsen und damit zu seiner Weiterentwicklung. In der gegenwärtigen Epoche jedoch strebt der Mensch eher danach, „mehr zu haben“, was im Grunde zu einer „moralischen Unterentwicklung“ führt. Der Mensch folgt dem Wohlstand und hält ihn für das wichtigste Ziel. Das bringt eine doppelte Möglichkeit: einerseits ist jener Wohlstand zur immer weiteren Entwicklung für den Menschen unerlässlich, andererseits jedoch schließt der Wohlstand den Menschen quasi wie in einem Gefängnis ein, wenn er als das höchste Gut gilt, außer dem man sich um nichts kümmern muss. Das Herz verhärtet sich, der Geist verschließt sich dem anderen, die Menschen kennen keine Freundschaft mehr, sondern nur noch das eigene Interesse ${ }^{31}$.

Ökonomische, gesellschaftliche und politische Entwicklung - so schreibt Benedikt XVI., sollte den Grundsatz der Selbstlosigkeit berücksichtigen, wenn sie authentisch menschlich sein will, also muss sie sich auf die Liebe, auf das Wohlergehen des anderen Menschen stützen. Entwicklung muss vor allem für den Menschen sein. All das trägt der Grundsatz der Gerechtigkeit in sich, dem der ganze Markt, der gegenseitige Austausch zwischen Rechtssubjekten, unterworfen ist; diese Gerechtigkeit steht ihnen alleine aufgrund ihrer Existenz zu. Die Gesellschaft ist ohne diesen Grundsatz keine Einheit, es fehlt an gesellschaftlichem Gleichgewicht und auch am Vertrauen zwischen den Bürgern. Das führt dazu, dass der Markt seine ökonomische Funktion nicht erfüllen kann: es beginnt die Dominanz des Stärkeren über den Schwächeren, es zeigt sich antagonistisches Verhalten, die „Vereitelung wahrer menschlicher Beziehungen" folgt, die das Funktionieren der Gesellschaft ernsthaft gefährdet.

Die grundlegende Zelle, aus der sich die Gesellschaft zusammensetzt und die ihre Entwicklung bedingt, ist die Familie. Die Ehe, die von Natur aus auf das Zeugen und Aufziehen von Nachkommen ausgerichtet ist,

\footnotetext{
${ }^{31}$ Vgl. Paul VI., Enzyklika Populorum progressio, Nr. 19.
} 
erfüllt eine wichtige Funktion für die Gesellschaft: Fortpflanzung - eine verantwortungsvolle Fortpflanzung, die einen Beitrag zur ganzheitlichen menschlichen Entwicklung leistet und neue Bürger hervorbringt. Der Papst unterstreicht in Populorum progressio, dass die natürliche Familie, in der „verschiedene Generationen zusammenleben und sich gegenseitig helfen, um zu größerer Weisheit zu gelangen und die Rechte der einzelnen Personen mit den anderen Notwendigkeiten des gesellschaftlichen Lebens zu vereinbaren, das Fundament der Gesellschaft ist" ${ }^{\text {“32 }}$. Eine moralisch verantwortungsvolle Offenheit für das Leben bildet einen gesellschaftlichen und ökonomischen Reichtum, deshalb sind die Pflege der Familie, Hilfe für die Familie, eine Bejahung des Familienlebens, das in den gegenwärtigen Zeiten viele seiner Werte und Bedeutungen verloren hat, wichtig. Heute, so zeigt sich, ist das Wesen von Ehe und Familie verdorben worden. All das führt zu einer demographischen Entvölkerung, denn der Parameter 1,4 enthüllt eine Wahrheit mit großer Durchschlagskraft: dass in solch einer Bevölkerung zukünftig die Verstorbenen nicht durch die Jungen ersetzt werden.

Eine Gesellschaft, die auf Glauben und Weisheit gebaut ist, ist eine gute Gesellschaft. Man kann Glauben weder vom öffentlichen Leben noch von Vernunft und Weisheit trenne, denn der Ausschluss der Religion aus der öffentlichen Sphäre entzieht dem menschlichen Leben Motivation und „die Vernunft bedarf stets der Reinigung durch den Glauben. [...] Die Religion bedarf ihrerseits stets der Reinigung durch die Vernunft, um ihr echtes menschliches Antlitz zu zeigen“"33. In diesem Kontext ist der Grundsatz der Subsidiarität der nächste, der unerlässlich für eine gute Gesellschaft ist. Papst Benedikt XVI. unterstreicht, dass sie Ausdruck menschlicher Freiheit ist, sie ist Hilfe für die Person „,durch die Autonomie der mittleren Gruppen und Verbände“", sie schätzt die Würde der Person und ist ausgerichtet auf die richtige menschliche Entwicklung, auf das Wohl des anderen Menschen. So wie die Liebe die Basis des Grundsatzes der Gerechtigkeit ist, so ist sie es auch bei diesem Grundsatz. Ohne Liebe existieren jene Grundsätze nicht, werden nicht in die Tat umgesetzt. Pater Jacek Salij unterstreicht in einer Reflexion zum Thema Patriotismus, der ebenfalls zum Aufbau einer Gesellschaft notwendig ist, dass das Vaterland zwar eine Gemeinschaft ist, die unterteilt ist nach Parteien, Gruppen, dass es aber trotz deren Konflikte

\footnotetext{
${ }^{32}$ Paul VI., Enzyklika Populorum progressio, Nr. 36.

${ }^{33}$ Benedykt XVI., Caritas in veritate, 2009, Nr. 56.
} 
und Aufeinanderprallen auf das gemeinsame Gute steuern muss. Man muss sich um die Gemeinschaft durch Hilfe für Schwächere und Benachteiligte kümmern. Man muss dazu beitragen, dass es im Land weniger Arbeitslosigkeit gibt. Man muss, besonders bei Kindern, Sensibilität für behinderte Menschen aufbauen und gesellschaftliche Pathologien überwinden ${ }^{34}$. All dieses Handeln beruht auf den Grundsätzen der Subsidiarität, und auch der Liebe und der Gerechtigkeit, die unerlässlich sind im menschlichen Leben. Der Aufbau einer guten Gesellschaft, die auf den Grundsätzen von Gerechtigkeit und Subsidiarität beruht, heißt auch, dass man sich um den Frieden kümmert, um die Konfliktverhütung, wobei man immer das gemeinsame Gute im Blick hat.

Die Liebe und die Weisheit sind in der Interpretation Benedikts XVI. die maßgeblichen Werte, die in einer guten Gesellschaft zum Ausdruck kommen, denn sie sind für die zwischenmenschlichen Beziehungen verantwortlich und diese wiederum implizieren ein angemessenes, richtiges Funktionieren der Gesellschaft. Liebe und Wahrheit, so könnte man sagen, sind die beiden Flügel der Zivilisation, ohne die man nicht leben kann und ohne die man die höchsten Werte nicht verkünden kann. Von der Gesellschaft hängt es ab, ob das vorhanden ist, was für den Menschen am wichtigsten ist, nämlich zu lieben und geliebt zu werden. Denn nur damit kann die Wirtschaft sich als gesellschaftliche Dimension durch den Menschen und für den Menschen entwickeln.

\section{Liebe und Wahrheit als Ausgangspunkt der Ökonomie}

Sich für das Leben zu öffnen ist von zentraler Bedeutung für die wirtschaftliche Entwicklung. Wenn die Gesellschaft zur Negation und Vereitelung des Lebens neigt, finden sich nicht die Motivation und Energie, die notwendig sind, um sich im Dienste des wahrhaften Guten für den Menschen $\mathrm{zu}$ engagieren. Wenn es zum Verlust persönlicher und gesellschaftlicher Sensibilität bei der Annahme neuen Lebens kommt, unterliegen auch andere Formen der Öffnung, die für das gesellschaftliche Leben nützlich sind, einem Erosionsprozess. Die Annahme des Lebens stärkt die moralischen Kräfte und befähigt zu gegenseitiger Hilfe. Wenn sie den Grundsatz der Öffnung für das

34 Vgl. J. Salij, Wychowanie do patriotyzmu, in: Autorytet prawdy. Wychowanie dzieci i młodzieży, M. Ryś (Hrsg.), Warszawa 2006, Verlag WSD, S. 93-95. 
Leben annehmen, können reiche Länder die Nöte der armen Länder besser verstehen, können die Nutzung riesiger wirtschaftlicher und intellektueller Ressourcen zur Befriedigung egoistischer Wünsche der eigenen Bürger vermeiden und edles Verhalten mit dem Gedanken an eine moralisch gesunde und solidarische Produktion unterstützen, unter Beachtung des fundamentalen Lebensrechts jedes Landes und jeder Person ${ }^{35}$.

Ein anderer Aspekt ist ein falscher Ökonomiebegriff. Menschliche Kosten sind immer auch ökonomische, und ein schlechtes Funktionieren der Wirtschaft zieht ebenfalls menschliche Kosten nach sich. Mit Blick auf die Menschenwürde und die Anforderungen der Gerechtigkeit sollten ökonomische Entscheidungen, besonders heute, nicht zu einer übermäßigen und moralisch nicht hinnehmbaren Vergrößerung der Unterschiede in der Verteilung des Reichtums führen ${ }^{36}$.

Die Enzyklika ruft zum Mut bei der Suche nach neuen Formen bei der Unterscheidung zwischen profit und non profit auf. Sie weist auf die Notwendigkeit hin, die Funktion des Staates und die Rolle der Gewerkschaften in der Epoche der Globalisierung neu zu durchdenken, damit diese sich wirklich um die Interessen ihrer Mitglieder kümmern und gleichzeitig gesellschaftliche Konsequenzen ihres Handelns für Gesellschaften in anderen Regionen der Welt im Auge behalten.

Die „Sünde“ der zeitgenössischen Ökonomie hängt damit zusammen, dass man sie nicht als eine Gesellschaftswissenschaft begreift, die die Schaffung von Gemeinwohl zum Ziel hat. Man hat begonnen, die Ökonomie als eine Art Maschine zur Gewinnvermehrung aufzufassen, die ,autonom gegenüber Werten“ ist. Man hat universelle Tugenden wie Anständigkeit, Sparsamkeit oder Verantwortung, auf denen der neuzeitliche Kapitalismus errichtet worden ist, vergessen. Der Papst weist darauf hin, dass für das Wirken des zeitgenössischen internationalen Kapitals letztlich niemand mehr die Verantwortung trägt: nicht der Staat und weder die Aktionäre, deren Beteiligungen weit gestreut sind, noch die Führungsriege der Manager, die nur an steigenden Kennzahlen interessiert ist. Das gesamte Handeln ist somit auf schnellen Ertrag ausgerichtet, was in der langfristigen Perspektive verhängnisvoll ist ${ }^{37}$. Man kann nicht leugnen, dass Kapital unter Umständen

\footnotetext{
${ }^{35}$ Vgl. J. Salij, Wychowanie do patriotyzmu, S. 93-95.

${ }^{36}$ Vgl. J. Salij, Wychowanie do patriotyzmu, S. 42.

37 Vgl. http://www.teziusz.pl (24 V 2011).
} 
auch Gutes erzielen kann, wenn es gut investiert wird. Man muss jedoch die Anforderungen der Gerechtigkeit respektieren und auch berücksichtigen, wie jenes Kapital überhaupt entstanden ist sowie die Schäden für andere Personen, die dadurch zustande kommen, dass das Kapital nicht an dem Ort eingesetzt wird, an dem es entstanden ist ${ }^{38}$. Bestimmte strukturelle Aspekte darf man weder verneinen noch verabsolutieren, denn die „Globalisierung ist a priori weder gut noch schlecht", sondern vor allem vom Menschen abhängig.

Das nächste Problem der Ökonomisierung ist der ,zunehmende Merkantilismus des kulturellen Austauschs“. Sein Ziel ist der „oft völlig unkritisch übernommene kulturelle Eklektizismus. Dieser Eklektizismus und eine relativistisch verstandene „kulturelle Angleichung“ führen nach Meinung des Papstes zu einem kulturellen Verfall und reduzieren den Menschen in der Konsequenz alleine auf ein kulturelles Faktum, was zur Unsicherheit einer „,neuen Art von Zwang oder Manipulation“ führt. Er warnt davor, Hilfe zu erteilen, bei der armen Ländern ein bestimmtes kulturelles Modell aufgezwungen wird. Als ein Beispiel erwähnt er „das Phänomen des internationalen Tourismus, der einen wesentlichen Faktor der ökonomischen Entwicklung und des kulturellen Wachstums bilden, sich bei Gelegenheit aber auch in Ausbeutung und moralischen Verfall verwandeln kann.“ Er kritisiert dabei das Vorgehen einiger internationaler Organisationen, die über einen riesigen bürokratischen Apparat verfügen und tatsächlich selbst den Löwenanteil der für die Hilfe vorgesehenen Mittel verschlingen. Deshalb fordert er schnelle Reformen bei diesen Organisationen ${ }^{39}$.

Die Überzeugung, sich selbst zu genügen und in der Lage zu sein, das in der Geschichte gegenwärtige Übel allein durch das eigene Handeln überwinden zu können, hat den Menschen dazu verleitet, das Glück und das Heil in immanenten Formen des materiellen Wohlstands und des sozialen Engagements zu sehen. Weiter hat die Überzeugung, dass die Wirtschaft Autonomie erfordert und keine moralische „Beeinflussung“ zulassen darf, den Menschen dazu gedrängt, das Werkzeug der Wirtschaft sogar auf zerstörerische Weise zu missbrauchen. Langfristig haben diese Überzeugungen zu wirtschaftlichen, gesellschaftlichen und politischen Systemen geführt, die die Freiheit der Person und der gesellschaftlichen Gruppen unterdrückt haben

\footnotetext{
38 Vgl. http://www.teziusz.pl (24 V 2011).

39 Vgl. http://www.teziusz.pl (24.05.2011).
} 
und genau aus diesem Grund nicht in der Lage waren, für die Gerechtigkeit zu sorgen, die sie versprochen hatten ${ }^{40}$.

Die Entwicklung braucht Christen, die die Arme zu Gott erheben in der Geste des Gebets, Christen, die von dem Bewusstsein getragen sind, dass die von Wahrheit erfüllte Liebe, caritas in veritate, von der die echte Entwicklung ausgeht, nicht unser Werk ist, sondern uns geschenkt wird. Darum müssen wir auch in den schwierigsten und kompliziertesten Angelegenheiten nicht nur bewusst reagieren, sondern uns vor allem auf Seine Liebe beziehen. Die Entwicklung beinhaltet Aufmerksamkeit für das geistliche Leben, ernsthafte Beachtung der Erfahrungen des Gottvertrauens, der geistlichen Brüderlichkeit in Christus, des Sich-Anvertrauens an die göttliche Vorsehung und Barmherzigkeit, der Liebe und Vergebung, des Selbstverzichts, der Annahme des Nächsten, der Gerechtigkeit und des Friedens ${ }^{41}$.

Die Ökonomie muss keineswegs zur Armut im Sinne von nicht vorhandenen Gütern führen. Deshalb erinnert Benedikt XVI. daran, dass eine der schlimmsten Varianten der Armut, die der Mensch erfahren kann, die Einsamkeit ist. Andere Formen der Armut, einschließlich materieller Armut, stammen aus der Isolation, daraus, dass man nicht geliebt wird oder Schwierigkeit hat, zu lieben, sie stammen aus der Zurückweisung der Liebe Gottes, aus dem primitiven, tragischen Verschließen des Menschen in sich selbst mit der Überzeugung, dass man sich selbst genügt oder dass man nur ein unbedeutender und vorübergehender Fakt ist, ein „Fremder“ im durch Zufall entstandenen Universum. Der Mensch ist entfremdet, wenn er alleine ist oder der Wirklichkeit entrissen, wenn er dem Denken und Glauben an ein gewisses Fundament aufgibt. So wie die familiäre Gemeinschaft keine der Personen ausschließt, aus denen sie sich zusammensetzt, und so wie die Kirche ganz und gar die neue Kreatur bejaht ${ }^{42}$, die sich durch die Taufe mit Seinem lebendigen Leib verbindet, so schließt auch die Einheit der menschlichen Familie keine Person, kein Land und keine Kultur aus sich aus, sondern macht sie noch reiner, noch verbundener miteinander in ihrer rechtmäßigen Vielfalt ${ }^{43}$.

\footnotetext{
${ }^{40}$ Vgl. Benedykt XVI., Caritas in veritate, 2009, Nr. 34.

${ }^{41}$ Vgl. Benedykt XVI., Caritas in veritate, 2009, Nr. 79.

${ }^{42}$ Vgl. Gal. 6,15; 2. Kor. 5,17.

${ }^{43}$ Vgl. A. Koprowski, Odważnie przeciw schematom, „Idziemy“ 28 (2009), S. 10-13.
} 
Für alle gibt es Platz auf dieser unserer Erde: auf ihr sollte die gesamte menschliche Familie den Reichtum finden, der für ein Leben in Würde notwendig ist, mit Hilfe der Natur selbst, die ein Geschenk Gottes für Seine Kinder ist und indem sie ihren eigenen Beitrag in Form von Arbeit und Erfindungsgeist einbringt. In vielen Lebenssituationen verbergen sich kulturelle Einstellungen, die die menschliche Würde negieren. Während die Elenden noch an die Türen der Reichen klopfen, tut die reiche Welt so, als höre sie das nicht, da ihr Gewissen schon nicht mehr in der Lage ist, zu erkennen, was Menschlichkeit bedeutet. Gott dagegen enthüllt vor dem Menschen die weite Welt, die auf den Pfeilern Verstand und Glauben ruht und auf diese Weise kann Er sich durch die Erkenntnis der Wahrheit denjenigen offenbaren, denen es an den Mitteln zum Leben mangelt. Im Verhalten des Menschen erfüllt sich somit der ökonomische Aspekt seines Daseins.

\section{Die Gesellschaft braucht geeignete Grundsätze}

Ohne Wahrheit, ohne Vertrauen und Liebe gegenüber dem Wahren gibt es kein Gewissen, keine soziale Verantwortung. Das soziale Handeln wird ein Spiel privater Interessen und Logiken der Macht, mit zersetzenden Folgen für die Gesellschaft, um so mehr in einer Gesellschaft auf dem Weg zur Globalisierung und in schwierigen Situationen wie der augenblicklichen ${ }^{44}$. Deshalb ist der wichtigste Grundsatz gemeinschaftlichen Lebens die Gerechtigkeit.

Nach dem Katechismus der Katholischen Kirche heißt das Respektieren von Menschenwürde und Menschenrechten. Weil die Person das Subjekt der Gesellschaft ist, deshalb sollte sie so handeln, dass Bedingungen geschaffen werden, die es jedem Menschen erlauben, das zu erreichen, was ihm zusteht, aufgrund der Tatsache seines Lebens und der persönlichen Dimension seiner ontischen Natur. Die Grundlagen dieser Ansicht haben Aristoteles und später Thomas von Aquin aufgestellt, der die Gerechtigkeit in drei Arten unterteilt hat (Tauschgerechtigkeit, austeilende Gerechtigkeit, Gesetzesgerechtigkeit).

Gerechtigkeit nimmt im gesellschaftlichen Leben zwei Sphären ein: die materielle und die geistige. Bei der materiellen geht es vor allem um das Recht auf Arbeit und gerechte Entlohnung, bei der geistigen um Respekt vor dem

\footnotetext{
${ }^{44}$ Vgl. Benedykt XVI., Caritas in veritate, 2009, Nr. 5.
} 
Menschen und seiner Würde. Die Quelle gesellschaftlicher Gerechtigkeit sind die menschlichen Einstellungen. Jede Person sollte sich der gleichen Würde und der gleichen Rechte erfreuen, denn sie ist geschaffen nach dem Bild Gottes, Gott ähnlich und besitzt einen verständigen Geist. Würden alle Bürger auf dieselbe richtige Weise gesellschaftliche Gerechtigkeit verstehen, würde sich unsere Gesellschaft ohne all die Probleme entwickeln, die gegenwärtig auftreten.

Der zweite Grundsatz ist das Gemeinwohl, das beschrieben wird als die Gesamtheit jener Bedingungen des gesellschaftlichen Lebens, die sowohl den Gruppen als auch den einzelnen Gliedern ein volleres und leichteres Erreichen der eigenen Vollendung ermöglichen ${ }^{45}$. Es geht um die Sorge für das Wohl des Individuums durch Engagement bei der Arbeit für das Gemeinwohl, also die Sorge um die Entwicklung des Menschen durch die Befriedigung grundlegender Bedürfnisse. Darüber hinaus geht es um Respekt und Unterstützung für die Menschenrechte der Person, um ein Wachstum der geistigen und irdischen Güter, des Friedens und der sozialen Sicherheit. Jeder Mensch sollte seiner Stellung und der von ihm ausgeübten Funktion entsprechend diese Rechte respektieren und sich um die Pflichten kümmern, für die er Verantwortung trägt; vor allem durch die Sorge um die eigene Familie, aufrichtiges Engagement bei der eigenen Arbeit und aktive Teilnahme am öffentlichen Leben. Vereinfachend könnte man sagen, man muss den anderen das geben, was sie dazu befähigt, ,ihre Flügel zu entfalten“.

Der nächste Grundsatz gesellschaftlichen Lebens ist der Grundsatz der gesellschaftlichen Solidarität. Sie ist verwurzelt in der Persönlichkeit und sozialen Natur des Menschen und verweist auf gegenseitige Verbindung und Pflicht, auf die gegenseitige Abhängigkeit zwischen Menschen und der Gesellschaft. Sie schließt Individualismus und Kollektivismus aus, der den Menschen im gesellschaftlichen Leben auf die Rolle eines Objektes reduziert. Durch gesellschaftliche Solidarität entsteht eine gewisse Würde im Handeln und Streben, im gegenseitigen Einverständnis und in gegenseitiger Unterstützung, eine wechselseitige Verantwortlichkeit für sich und den anderen Menschen. Sie beruht nicht auf Mitleid, sondern auf ehrlichem Interesse für den anderen, für seine Probleme und Bedürfnisse. Anhand literarischer Beispiele lernen wir von Kindesbeinen an beispielsweise den Grundsatz „Einer für alle und alle für einen“. Dem könnte man noch den Grundsatz „Jeder für jeden“ hinzufügen und es kommt ein vollständiges Bild 
der Grundlagen von Solidarität zum Vorschein, die eine gute Gesellschaft bildet und ihre Entwicklung stimuliert.

Noch eine weitere Grundlage des Lebens in einer Gesellschaft ist das Prinzip der Subsidiarität. Dabei geht es darum, dass größere gesellschaftliche Organe und organisierte öffentliche Institutionen den Individuen und kleineren Gruppen durch ihr Handeln helfen und sie ergänzen. $\mathrm{Zu}$ unterstreichen sind hierbei gegenseitige Pflicht und Schuldigkeit sowie die Verbindungen zwischen Individuum einerseits und Gruppen andererseits, die für eine gute bürgerliche Gesellschaft wesentlich sind. Dabei ist noch eine weitere Wahrheit über das Funktionieren einer Gesellschaft bedeutsam, nämlich staatliche Hilfe für die bedürftigsten Personen. Jeder, der ein Problem hat, kann sich mit der Bitte um Hilfe an den Staat wenden. Das zeigt auch, dass der Mensch das eigentliche Ziel, das Subjekt des sozialen Lebens ist.

Die letzte, aber nicht minder wichtige Grundlage für gesellschaftliches Leben ist die Liebe. Nichts sichert den Frieden und die Ordnung auf der Welt so vollständig und so zuverlässig wie die Liebe. Würde die Welt von der Liebe verlassen, versagten alle Vorschriften und Gesetze und der Mensch fände sich in der Wirklichkeit nicht mehr wieder. Fehlte es an Selbstlosigkeit, an uneigennützigem Geben und an Liebe ohne Erwartung einer Kompensation, wäre das Leben des Menschen traurig und eher dem Rückschritt als dem Fortschritt bestimmt. Der Grundsatz „caritas“ ist eine natürliche Tugend; somit ist die Pflicht jedes Menschen, der sich ernsthaft mit der Schaffung einer Zivilisation der Liebe befasst, die Brüderschaft in Christus. Gott ist die Quelle der „caritas“, von Ihm stammt unser Leben und auf Ihn ist es gerichtet. Er ist der Adressat unseres Handelns, Er gibt uns Kraft, Herausforderungen, Pflichten und Aufgaben im täglichen Leben anzunehmen. Gerade diese Liebe in der Gesellschaft ergänzt sich komplementär mit der Gerechtigkeit und führt uns zur Wahrheit der menschlichen Existenz.

\section{Schlussfolgerung}

Caritas in veritate beschäftigt sich mit der Gesellschaft, ist aber zugleich ein zutiefst theologisches Dokument. Die letzten Jahrzehnte waren eine Zeit bedeutender Verzerrungen, um nicht zu sagen Zerstörung der Kontinuität kultureller Überlieferung. Der „katholische Wortschatz“ ist für viele nicht mehr selbstverständlicher Träger von Inhalten, die man ernst nehmen muss. 
Währenddessen empfangen wir die Früchte der Überlegungen des Papstes, und auch die Erfahrungen, die die Kirche, die wahrhaft universelle Kirche, in sehr unterschiedlichen Kontexten sammelt, in Ländern, Kontinenten, Nationen und bei der Arbeit internationaler Organisationen in den Zeiten der Globalisierung ${ }^{46}$.

Damit eine Gesellschaft richtig funktionieren kann, braucht man auch im ökonomischen Bereich Menschen, die einen rechtschaffenen Charakter haben. Wer Christ ist, sollte stets zu diesen gehören, ohne Rücksicht auf verschiedene Überzeugungen, die in der Gesellschaft lanciert werden. Gegenwärtig kann man eine Dichotomie bemerken, eine Trennung zwischen dem Privatleben und dem gesellschaftlichen und politischen Leben. Es gibt Leute, die genau diese Trennung fordern, denn ihr Privatleben sei doch ausschließlich ihre eigene Sache. Dazu lohnt es sich, einige Thesen aufzustellen. Wenn jemand nicht in der Lage ist, für seine eigene Familie zu sorgen, wird er sich dann objektiv um andere Familien in Polen kümmern? Kann jemand, der ein Alkoholproblem hat, ein Vorbild für Kinder und Jugendliche sein? Wird jemand, der seinen Kindern kein guter Vater ist, der Gesellschaft ein guter Vater sein? Fragen ähnlicher Art könnte man Tausende stellen. Die Gestaltung einer Gesellschaft hängt davon $a b$, dass ein jeder von uns sich dieser Zusammenhänge bewusst wird und von sich aus die am Besten geeigneten Personen wählen und von ihnen repräsentiert werden will. Heute kann man leider feststellen, dass Menschen mit klaren moralischen Grundsätzen und reinem Gewissen Intoleranz entgegenschlägt. Solche Leute, die oft bescheiden und nicht in der Lage sind, sich für ihre Standpunkte einzusetzen, werden nicht nur an den Rand der Gesellschaft gedrängt, sondern allzu oft diskriminiert. In den Massenmedien wird rechtschaffenen Menschen gegenwärtig eine Art Scheintoleranz eingeräumt, obwohl doch diese die Bedingungen schaffen sollten, die eine richtige Entwicklung der Gesellschaft ermöglichen.

Caritas in veritate als Kern einer guten Gesellschaft sollte Einfluss haben auf ihre ganzheitliche Entwicklung. Integration im Inneren ist, nach der Soziologie, eine Eigenschaft einer funktionierenden Gesellschaft. Jeder in der Gesellschaft erfüllt eine bestimmte Rolle bzw. Funktion. Auf diese Art und Weise ist die Gesellschaftunterteilt, aberdieseUnterteilung soll eine ganzheitliche Gesamtheit schaffen, in der die Rollen sich komplementär ergänzen, eine bedingt die andere: so existiert eine gute, integrierte Gesellschaft. Dieser Abhängigkeit einer Person von der anderen sollten die Regierungen der Staaten Aufmerksamkeit

${ }^{46}$ Vgl. A. Koprowski, Odważnie przeciw schematom, „Idziemy“ 28 (2009), S. 13. 
schenken, um ihr Unterstützung und Hilfe zu gewähren. Vor allem aber sollten sie einen Beitrag zur Verbreitung derartiger Verhaltensweisen leisten, damit trotz der „Evolution“ der Gesellschaft diese Integration fortbestehen kann.

Liebe und Wahrheit sind zwei Attribute einer guten Gesellschaft, mit denen jeder Mensch ausgestattet ist. Von Anbeginn der Zeiten, von Beginn der menschlichen Existenz an, ist der Mensch dazu berufen, sich von Liebe und Wahrheit leiten zu lassen. $\mathrm{Zu}$ dieser Wahrheit ruft ihn das christliche Leben bzw. die ontische Dimension seiner Natur auf. Eine Gesellschaft ohne Liebe und Wahrheit ist von Agonie bedroht, denn sie hört nicht nur auf, sich zu entwickeln, sondern ihr auf Irrtum beruhender ökonomischer Status führt sie geradewegs ins Nichts. Daher muss man ohne jeden Zweifel die These annehmen, dass sich, damit die Gesellschaft gut wird, der homo economicus im Namen von Liebe und Wahrheit in den homo religiosus verwandeln muss.

\section{Bibliography}

Benedykt XVI., Caritas in veritate, Tarnów 2009, Verlag Biblos.

Filipiak M., Socjologia kultury, Zarys zagadnień, Lublin 2003, Verlag UMCS.

Frankowski M. T., Socjologia. Makrostruktury społeczne, Warszawa 2008, Verlag M.M.

Kowalik S., Szkic o koncepcjach socjalizacji, in: Edukacja wobec zmiany społecznej,

J. Brzezinski, L. Witkowski (Hrsg.), Poznań-Toruń 1994, Verlag Edytor.

Mariański J., Młodzież między tradycją a ponowoczesnościa, Lublin 1995, Verlag KUL.

Młyński J., Kościół Polski wobec problemów społecznych w świadomości abiturientów Tarnowa, „Tarnowskie Studia Teologiczne” XXVI/2 (2007), p. 89-107.

Modzelowski W., Wprowadzenie do socjologii, Katowice 1999, Verlag BPS Śląsk.

Ogburn W. P., Nimkoff W. F., Handbook of Sociology, London 1947.

Paleszczuk J., Czym jest społeczeństwo, in: Wymiary życia społecznego. Polska na przełomie XX i XXI wieku, M. Marody (Hrsg.), Warszawa 2007, Verlag Scholar.

Stala J., Katecheza o matżeństwie i rodzinie w Polsce po Soborze Watykańskim II, Tarnów 2004, Verlag Biblos.

Stala J., Katecheza rodzinna. Próba teologicznego zarysu zagadnienia, in: Rodzina. Bezcenny dar i zadanie, J. Stala, E. Osewska (Hrsg.), Radom 2006, Verlag Polwen.

Szczepański J., Socjologia, Rozwój problematyki i metod, Warszawa 1961, Verlag Żak.

Turner J., Struktura teorii socjologicznej, Warszawa 1985, Verlag PWN.

Weber M., Gospodarka i społeczeństwo. Zarys socjologii rozumiejacej, Warszawa 2002, Verlag PWN.

Wymiary życia społecznego Polska na przełomie XX i XXI wieku, M. Marody (Hrsg.), Warszawa 2007, Verlag Scholar. 